\title{
High Weight Loss during Radiation Treatment Changes the Prognosis in Under-/Normal Weight Nasopharyngeal Carcinoma Patients for the Worse: A Retrospective Analysis of 2433 Cases
}

\author{
Lu-Jun Shen ${ }^{1,39}$, Chen Chen ${ }^{1,39}$, Bo-Fei Li ${ }^{1,3}$, Jin Gao ${ }^{1,2}$, Yun-Fei Xia ${ }^{1,2 *}$ \\ 1 State Key Laboratory of Oncology in Southern China, Cancer Center, Sun Yat-sen University, Guangzhou, People's Republic of China, 2 Department of Radiation \\ Oncology, Cancer Center, Sun Yat-sen University, Guangzhou, People's Republic of China, $\mathbf{3}$ Zhongshan School of Medicine, Sun Yat-sen University, Guangzhou, People's \\ Republic of China
}

\begin{abstract}
Background: Although weight loss is common in nasopharyngeal carcinoma (NPC) patients receiving radiotherapy, the prognostic influence of weight loss and its impact modified by body mass index (BMI) are still unclear.

Methods: 2433 NPC patients receiving radical radiotherapy at Sun Yat-sen University Cancer Center from November, 2000 to December, 2004 were enrolled. Weight change during radiation treatment was categorized into high weight loss (HWL) and low weight loss (LWL). The associations of HWL with overall survival (OS) and disease-specific survival (DSS) were analyzed by Cox regression.

Results: Among underweight patients, HWL was independently associated with poor OS (hazard ratio [HR], 2.06; $95 \% \mathrm{Cl}$ 1.36-3.11) and DSS (HR, 2.27; 95\% Cl 1.38-3.73), as compared with LWL, after adjusting for covariates. In normal weight patients, the impact of HWL on OS (HR, 1.47; 95\% Cl 1.19-1.80) and DSS (HR, 1.59; 95\% Cl 1.24-2.03) was moderate. Among overweight/obese patients, no significant association between HWL and OS (HR, 1.22; 95\% Cl 0.95-1.55), or DSS (HR, 1.23; $95 \% \mathrm{Cl} 0.93-1.64)$ was found.

Conclusion: Except for overweight/obese patients, high weight loss during radiation treatment was independently associated with poor survival in NPC. This impact was more prominent in the underweight patient group.

Citation: Shen L-J, Chen C, Li B-F, Gao J, Xia Y-F (2013) High Weight Loss during Radiation Treatment Changes the Prognosis in Under-/Normal Weight Nasopharyngeal Carcinoma Patients for the Worse: A Retrospective Analysis of 2433 Cases. PLoS ONE 8(7): e68660. doi:10.1371/journal.pone.0068660

Editor: Gayle E. Woloschak, Northwestern University Feinberg School of Medicine, United States of America

Received October 18, 2012; Accepted May 31, 2013; Published July 15, 2013

Copyright: (c) 2013 Shen et al. This is an open-access article distributed under the terms of the Creative Commons Attribution License, which permits unrestricted use, distribution, and reproduction in any medium, provided the original author and source are credited.

Funding: This work was supported by grants from the Hi-Tech Research and Development Program of China (No. 2006AA02Z4B4), and National Natural Science Foundation of China (No. 30770641; No. 31170805). The funders had no role in study design, data collection and analysis, decision to publish, or preparation of the manuscript.

Competing Interests: The authors have declared that no competing interests exist.

*E-mail: xiayf@sysucc.org.cn

9 These authors contributed equally to this work.
\end{abstract}

\section{Introduction}

Nasopharyngeal carcinoma (NPG) is an endemic head and neck epithelial malignancy. Approximately $60 \%$ patients with nasopharyngeal carcinoma present stage III or IV disease at initial diagnosis [1,2]. Although the treatment effect of NPG has improved primarily due to the progress in diagnostic imaging, radiation techniques and chemotherapy regimens, $20-30 \%$ of the patients will die due to cancer recurrence or/and distant metastasis [3-5]. Identification of those high-risk patients may provide new clues in developing clinical intervention to improve their survival.

Body mass index (BMI), a commonly utilized measure for indicating nutritional status in adults, has been shown closely associated with the prognosis of NPG patients [6,7]. Various studies have focused on the striking association between low BMI at diagnosis and poor prognosis due to higher local-regional recurrence and mortality. As emphasized by Shen et al., the hazard ratio for death was 0.66 for overweight $(95 \%$ CI, 0.48 to 0.90 ) and 0.47 (95\% CI, 0.23 to 0.97 ) for obese patients comparing to the baseline of normal weight or underweight patients [7].

In contrast to the considerable amount of research on BMI, the impact of body weight loss on NPG recurrence and death has not been addressed. More than $60 \%$ of NPC patients receiving curative-intent radiotherapy suffered from a weight loss greater than $5 \%$ during the treatment $[8,9]$. Advanced tumors, concurrent chemotherapy, and high BMI are important predisposing factors of weight loss, and it is reported that a critical weight loss $(>5 \%)$ during the radiation was associated with poorer treatment tolerance and worse prognosis in head and neck cancer (HNC) patients $[10,11]$.

Is weight loss also an unfavorable prognostic factor for NPC? Since BMI affects both weight loss and survival among NPC 
patients [9], analysis combining these two weight-related factors may be required. Therefore, in this study, we investigated the prognostic influence of weight loss and its corresponding potential effect-modification by BMI.

\section{Patients and Methods}

\section{Patients}

The medical records of 2820 newly diagnosed NPC patients without distant metastasis in Sun Yat-sen University Cancer Center (SYSUCG) from November, 2000 to December, 2004 were reviewed. Sun Yat-sen University Cancer Center Hospital Ethics Committee approved this study (No. YP201012). This was a retrospective analysis of routine data and therefore we requested and were granted a waiver of individual informed consent from the ethics committee. The data were collected by trained SYSUCC interviewers and analyzed anonymously. All patients received radical radiotherapy and completed the prescribed course of treatment. Exclusion criteria are any of the following: (i) missing weight measurement at baseline and/or at the end of radiotherapy (200); (ii) follow-up period less than 5 years (178); (iii) age less than 18 years old (9). A total of 2433 patients were enrolled. All patients received routinely nasopharyngeal CT or MRI examination before treatment and were staged according to the sixth edition of UICG staging system [12].

\section{Measurement and Grouping}

Pre-radiation treatment (pre-RT) weight was measured within 7 days before radiotherapy (RT), and post-radiation treatment (postRT) weight was measured within 7 days after completion of RT. BMI was defined as pre-RT weight $(\mathrm{kg})$ divided by the square of height (meter) and was categorized according to the WHO recommendations for Asian population [13]; because the proportion of obese patients was relatively small $(6.5 \%)$, we merged overweight and obese patients and obtained three BMI groups: $<18.5 \mathrm{~kg} / \mathrm{m}^{2}$, underweight, UW; $18.5-<23.0 \mathrm{~kg} / \mathrm{m}^{2}$, normal weight, NW; $\geq 23.0 \mathrm{~kg} / \mathrm{m}^{2}$, overweight/obese, OW (Figure 1).

Weight loss $(\Delta \mathrm{W})$ was calculated as the relative percent of weight changes between weight measurement before and after the radiation treatment,

$$
\left(\Delta W=\frac{W_{\text {pre }-R T} W_{\text {post }-R T}}{W_{\text {pre }-R T}} \times 100 \%\right)
$$

A number of studies suggest that a greater than $5 \%$ body weight loss during radiation for HNC patients indicates malnutrition and is clinically meaningful $[9,14,15] . \Delta \mathrm{W}$ was accordingly categorized into high weight loss $(\mathrm{HWL} ; \geq 5 \%)$ and low weight loss (LWL; $<5 \%)$.

\section{Treatment}

Radiotherapy included two-dimensional conformal radiotherapy (2D-GRT), three-dimensional conformal radiotherapy (3DCRT), and intensity-modulated radiotherapy (IMRT). Opposing lateral facial-cervical fields were used in the 2D-CRT to cover the nasopharynx and upper cervical lymphatic drainage region, with 1 lower anterior cervical field to cover the lower cervical region. After 36 to $40 \mathrm{~Gy}$, opposing lateral preauricular fields were used for the primary region, and anterior split neck fields were used for the cervical region. The primary tumor was irradiated to a dose of 60 to $78 \mathrm{~Gy}$. The dose of prophylactic irradiation for patients with 2D-CRT was 50-54 Gy to the prophylactic areas. For 3D-CRT, the total prescribed dose was 66-72 Gy to the gross tumor volume of nasopharynx (GTVnx), 60 to 70 Gy to the region involved by the metastatic lymph nodes (GTVnd), 60 Gy to high risk microscopic (CTV1, the GTVnx and an additional 5- to 10-mm margin), and 50-54 Gy to the low risk microscopic (CTV2). For IMRT, the target definition and delineation were the same as for 3D-CRT. The prescription dose was 68 Gy to the GTVnx, 60 to 64 Gy to the GTVnd of neck, 60 Gy to the GTV1, and 54 Gy to CTV2.

Chemotherapy included induction chemotherapy, concomitant chemotherapy, and adjuvant chemotherapy. The induction or adjuvant chemotherapy regimen was fluorouracil (5-Fu) plus cisplatin. The concurrent chemotherapy regimens were either 5Fu plus cisplatin or cisplatin alone; the 5 -Fu plus cisplatin regimen was 70 to $100 \mathrm{mg} / \mathrm{m}^{2}$ of cisplatin on day 1 plus 500 to $750 \mathrm{mg} / \mathrm{m}^{2}$ 5 -Fu on day 2 till day 5 every 3-4 weeks, for 2 to 3 cycles, and the cisplatin regimen was 30 to $40 \mathrm{mg} / \mathrm{m}^{2}$ of cisplatin every week, for 6 to 7 cycles.

\section{Follow-up and End Points}

After completion of RT, patients were followed up every 3 months for the first 3 years by clinic visits, telephone, letter and so on. The intervals gradually increased to $6-12$ months after 3 years. The data of follow-up was last reviewed on February 2011.

The primary outcomes were overall survival (OS) and diseasespecific survival (DSS). Secondary outcomes were local-regional recurrence free survival (LRFS) and distant metastasis free survival (DMFS). OS was defined as time from diagnosis to death by any causes. DSS was defined as the time from the start of RT to death caused by disease progression or treatment-related complications. LRFS was defined as time from the start of RT to the first occurrence of locoregional failure or death from the primary cancer without a documented site of recurrence or metastasis.

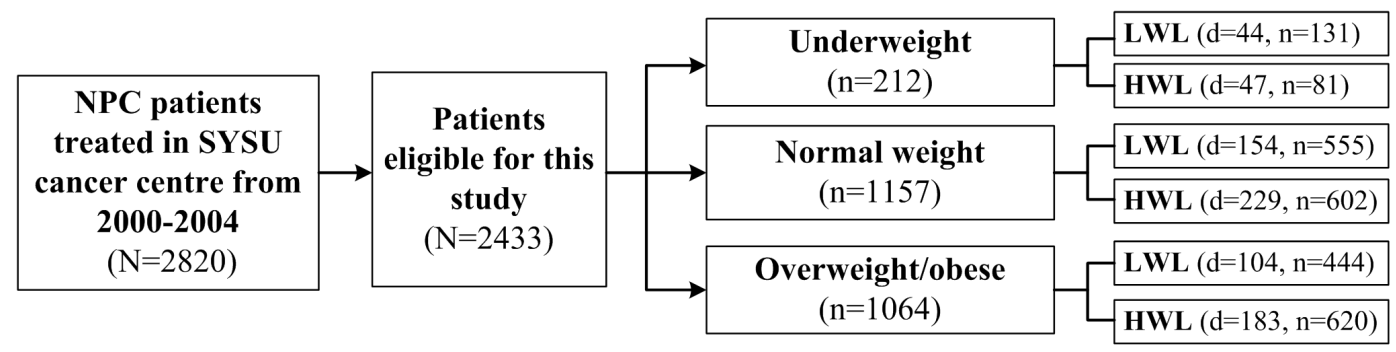

Figure 1. Flowchart of study design. A total of 2433 patients met the enrollment criteria. The population were first divided into $2 \times 3$ subgroups by BMI levels and weight loss status, and analyzed in the multiple-adjusted Cox model. Stratified analysis by BMI levels was further conducted to determine the impact of high weight loss during treatment for NPC patients with different BMI levels. Footnote: $d$, death; $n$, number of patients. doi:10.1371/journal.pone.0068660.g001 
Table 1. Baseline characteristics by weight loss status in patients with nasopharyngeal carcinoma $(n=2433)$.

\begin{tabular}{|c|c|c|c|c|c|c|c|}
\hline \multirow{3}{*}{$\begin{array}{l}\text { Patient characteristics } \\
\text { Duration of RT } \\
\text { (days, mean } \pm \text { SD) }\end{array}$} & \multirow{2}{*}{\multicolumn{2}{|c|}{$\begin{array}{l}\text { All } \\
51.0 \pm 6.3\end{array}$}} & \multirow{2}{*}{\multicolumn{2}{|c|}{$\begin{array}{l}\text { LWL } \\
50.5 \pm 5.9\end{array}$}} & \multirow{2}{*}{\multicolumn{2}{|c|}{$\frac{H W L}{51.4 \pm 6.5}$}} & \multirow{3}{*}{$\begin{array}{l}P \\
0.072^{\Psi}\end{array}$} \\
\hline & & & & & & & \\
\hline & No. & $\%$ & No. & $\%$ & No. & $\%$ & \\
\hline Age, years & & & & & & & 0.210 \\
\hline$<46$ & 1285 & 52.8 & 613 & 54.2 & 672 & 51.6 & \\
\hline$\geq 46$ & 1148 & 47.2 & 518 & 45.8 & 630 & 48.4 & \\
\hline Gender & & & & & & & 0.099 \\
\hline Male & 1851 & 76.1 & 877 & 77.6 & 974 & 74.8 & \\
\hline Female & 582 & 23.9 & 253 & 22.4 & 329 & 25.2 & \\
\hline UICC Stage & & & & & & & $<0.001$ \\
\hline 1 & 127 & 5.2 & 80 & 7.1 & 47 & 3.6 & \\
\hline$\|$ & 856 & 35.2 & 425 & 37.6 & 431 & 33.1 & \\
\hline III & 979 & 40.2 & 430 & 38.1 & 549 & 42.1 & \\
\hline IV & 471 & 19.4 & 195 & 17.3 & 276 & 21.2 & \\
\hline UICC T Stage & & & & & & & 0.037 \\
\hline $\mathrm{T} 1$ & 395 & 16.2 & 204 & 18.1 & 191 & 14.7 & \\
\hline $\mathrm{T} 2$ & 1023 & 42.0 & 485 & 42.9 & 538 & 41.3 & \\
\hline T3 & 621 & 25.5 & 273 & 24.2 & 348 & 26.7 & \\
\hline T4 & 394 & 16.2 & 168 & 14.9 & 226 & 17.3 & \\
\hline UICC N Stage & & & & & & & $<0.001$ \\
\hline No & 635 & 26.1 & 354 & 31.3 & 281 & 21.6 & \\
\hline $\mathrm{N} 1$ & 976 & 40.1 & 439 & 38.8 & 537 & 41.2 & \\
\hline N2 & 732 & 30.1 & 303 & 26.8 & 429 & 32.9 & \\
\hline N3 & 90 & 3.7 & 34 & 3.0 & 56 & 4.3 & \\
\hline BMI & & & & & & & $<0.001$ \\
\hline Underweight & 212 & 8.7 & 131 & 11.6 & 81 & 6.2 & \\
\hline Normal weight & 1157 & 47.6 & 555 & 49.1 & 602 & 46.2 & \\
\hline Overweight/obese & 1064 & 43.7 & 444 & 39.3 & 620 & 47.6 & \\
\hline Treatment & & & & & & & $<0.001$ \\
\hline RT & 1088 & 44.7 & 579 & 51.2 & 509 & 39.1 & \\
\hline $\mathrm{RT}+\mathrm{CT}$ & 1345 & 55.3 & 551 & 48.8 & 794 & 60.9 & \\
\hline
\end{tabular}

Footnote: UICC, International Union Against Cancer; BMI, body mass index; RT, radiotherapy; CT, chemotherapy; LWL, low weight loss; HWL, high weight loss; $\Psi$, result of t-test; $\mathrm{P}<0.05$ was considered statistically significant.

doi:10.1371/journal.pone.0068660.t001

DMFS was defined as time from the start of RT to the first occurrence of distant failure.

\section{Statistical Analysis}

Wilcoxon rank sum and Chi-square test were used to compare ordinal and categorical variables between groups, respectively. Rates of DSS, OS, LRFS, and DMFS were estimated by means of the Kaplan-Meier method and were compared between the subgroups with the use of the log-rank test. Cox regression model including the two main effect parameters (weight loss and BMI; in categorical form) and their interaction effect parameters was utilized to test the interaction effect between weight loss status and BMI levels. The multiple-adjusted Cox regression model was used to evaluate the survival of $2 \times 3$ weight-based subgroups divided by weight loss status (HWL vs LWL) and BMI levels (UW vs NW vs OW), with the covariates including age, sex, UICG T stage, UICG $\mathrm{N}$ stage, and treatment (with or without chemotherapy) [16,17]; the $2 \times 3$ subgroups were as follows: NW+LWL (group 1, reference), NW+HWL (group 2), UW+LWL (group 3), $\mathrm{UW}+\mathrm{HWL}$ (group 4), OW+LWL (group 5), and OW+HWL (group 6). Stratified multiple-adjusted Cox model by BMI levels was further conducted to evaluate the prognostic significance of weight loss status in each BMI group. A $\mathrm{P}$ value $<0.05$ was considered significant. Statistical analysis was performed using SPSS 20.0 software.

\section{Results}

\section{Characteristics of the Population}

Listed in Table 1 were baseline characteristics of patients. The median age was 46 years (range, 18-78 years). Among all the patients, $2166(89.0 \%)$ had undifferentiated non-keratinizing carcinoma, $203(8.3 \%)$ had differentiated non-keratinizing carcinoma and $64(2.6 \%)$ had other types; $983(40.4 \%)$ had early-stage disease (stage I/II), and $1450(59.6 \%)$ had advanced disease (stage III/IV). All patients received definitive-intent radiotherapy during 
Table 2. Unadjusted analysis for 5-year OS, DSS, LRFS, and DMFS rates.

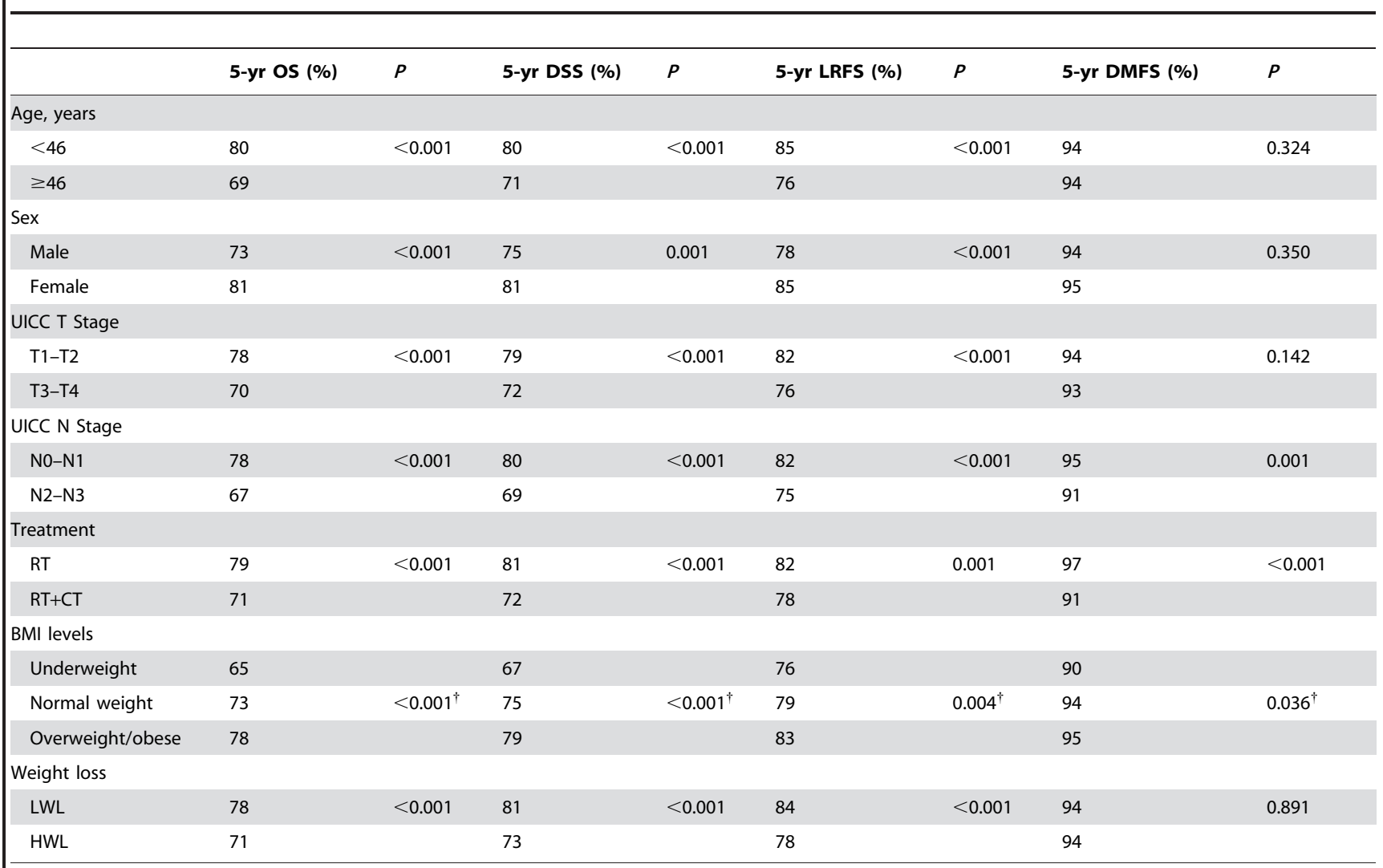

Footnote: OS, overall survival; DSS, disease-specific survival; LRFS, local-regional recurrent free survival; DMFS, distant metastasis free survival. Other abbreviation as in Table 1;

†result of joint test.

doi:10.1371/journal.pone.0068660.t002

treatment, with $2194(90.2 \%)$ patients treated with 2D-CRT, 63 $(2.6 \%)$ patients with 3D-CRT, and $176(7.2 \%)$ patients with IMRT. The duration of RT ranged from 42 to 90 days (median, 50 days). Induction chemotherapy, concurrent chemotherapy and adjuvant chemotherapy was delivered in 830 (34.1\%), $504(20.7 \%)$

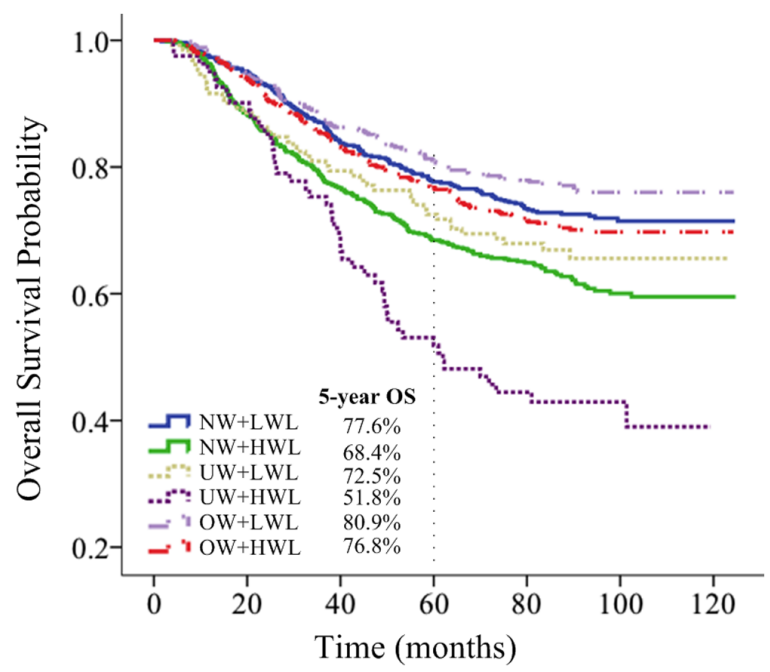

Figure 2. OS for NPC patients after radical radiotherapy in $2 \times 3$ grouping by BMI levels and weight loss status. doi:10.1371/journal.pone.0068660.g002 and $11(0.5 \%)$ patients, respectively. The median duration of follow-up was 85 months (range, 2-125 months).

For the entire population, the mean BMI was $22.60 \mathrm{~kg} / \mathrm{m}^{2}$. A total of 212 patients $(8.7 \%)$ were underweight, $1157(47.6 \%)$ were normal weight, and 1064 (43.7\%) were overweight/obese. During radiation treatment, the mean weight loss was $4.33 \mathrm{~kg}$, and $53.6 \%$ (1303) patients had high weight loss (HWL; $\Delta \mathrm{W} \geq 5 \%$ ). The proportion of HWL was higher in patients with advanced $\mathrm{T}$ stage, $\mathrm{N}$ stage, high BMI level and who received chemotherapy. No significant differences in age and sex were found across the weight loss subgroups (Table 1).

\section{BMI, Weight Loss, and Survival}

$572(23.5 \%)$ of the 2433 patients experienced cancer recurrence, $166(6.8 \%)$ had distant metastasis, and $761(31.3 \%)$ died. The 1-, 3-, and 5-year overall survival (OS) rates for the whole population were $96.8 \%, 83.8 \%$, and $74.6 \%$, respectively.

In unadjusted analysis, underweight patients had significantly lower 5 -year OS rate compared to normal weight $65 \%$ vs $73 \%$, $\mathrm{P}=0.004)$ and overweight/obese patients $(65 \%$ vs $78 \%, \mathrm{P}<0.001)$; HWL patients presented with significantly lower 5-year OS rate compared to LWL patients $(\mathrm{P}<0.001)$. Age, sex, UICC T stage, UICG $\mathrm{N}$ stage, and treatment also significantly influenced OS $(\mathrm{P}<0.001$ for all). Results on 5-year DSS and LRFS were similar to that of OS, while no difference in DMFS was found across the weight loss groups (Table 2).

The BMI $\times$ Weight loss interaction terms were tested for OS, and a borderline significance was identified for the differential 
Table 3. Multiple-adjusted analysis for OS and DSS among the whole population $(n=2433)$.

\begin{tabular}{|c|c|c|c|c|c|c|}
\hline \multirow{2}{*}{ Variables } & \multicolumn{3}{|l|}{ os } & \multicolumn{3}{|l|}{ DSS } \\
\hline & HR & $95 \% \mathrm{Cl}$ & $\mathbf{P}$ & HR & $95 \% \mathrm{Cl}$ & $\mathbf{P}$ \\
\hline Age, years & & & $<0.001$ & & & $<0.001$ \\
\hline$<46$ & Ref & & & Ref & & \\
\hline$\geq 46$ & 1.83 & $1.58-2.12$ & & 1.65 & $1.40-1.96$ & \\
\hline Sex & & & $<0.001$ & & & $<0.001$ \\
\hline Male & Ref & & & Ref & & \\
\hline Female & 0.68 & $0.57-0.82$ & & 0.69 & $0.56-0.86$ & \\
\hline UICC T Stage & & & $<0.001$ & & & $<0.001$ \\
\hline T1-T2 & Ref & & & Ref & & \\
\hline T3-T4 & 1.35 & $1.17-1.57$ & & 1.34 & $1.13-1.59$ & \\
\hline UICC N Stage & & & $<0.001$ & & & $<0.001$ \\
\hline N0-N1 & Ref & & & Ref & & \\
\hline N2-N3 & 1.44 & $1.23-1.67$ & & 1.45 & $1.21-1.74$ & \\
\hline Treatment & & & 0.049 & & & 0.064 \\
\hline $\mathrm{RT}$ & Ref & & & Ref & & \\
\hline $\mathrm{RT}+\mathrm{CT}$ & 1.18 & $1.00-1.38$ & & 1.23 & $1.02-1.49$ & \\
\hline $\begin{array}{l}\text { BMI+Weight loss } \\
(2 \times 3 \text { groups })\end{array}$ & & & $<0.001^{\dagger}$ & & & $<0.001^{\dagger}$ \\
\hline NW+LWL & Ref & & & Ref & & \\
\hline $\mathrm{NW}+\mathrm{HWL}$ & 1.45 & $1.18-1.78$ & $<0.001$ & 1.57 & $1.22-2.01$ & $<0.001$ \\
\hline UW+LWL & 1.25 & $0.89-1.75$ & 0.191 & 1.23 & $0.80-1.88$ & 0.348 \\
\hline UW+HWL & 2.56 & $1.65-3.56$ & $<0.001$ & 2.66 & $1.82-3.88$ & $<0.001$ \\
\hline$O W+L W L$ & 0.82 & $0.64-1.05$ & 0.116 & 0.90 & $0.67-1.22$ & 0.501 \\
\hline $\mathrm{OW}+\mathrm{HWL}$ & 0.98 & $0.79-1.22$ & 0.881 & 1.08 & $0.83-1.40$ & 0.577 \\
\hline
\end{tabular}

Footnote: Ref, reference; $\mathrm{HR}$, hazard ratio.

†result of joint test.

doi:10.1371/journal.pone.0068660.t003

impact of weight loss between underweight and overweight/obese patients (normal weight $\times$ HWL vs underweight $\times$ LWL, $\mathrm{P}=0.239$;
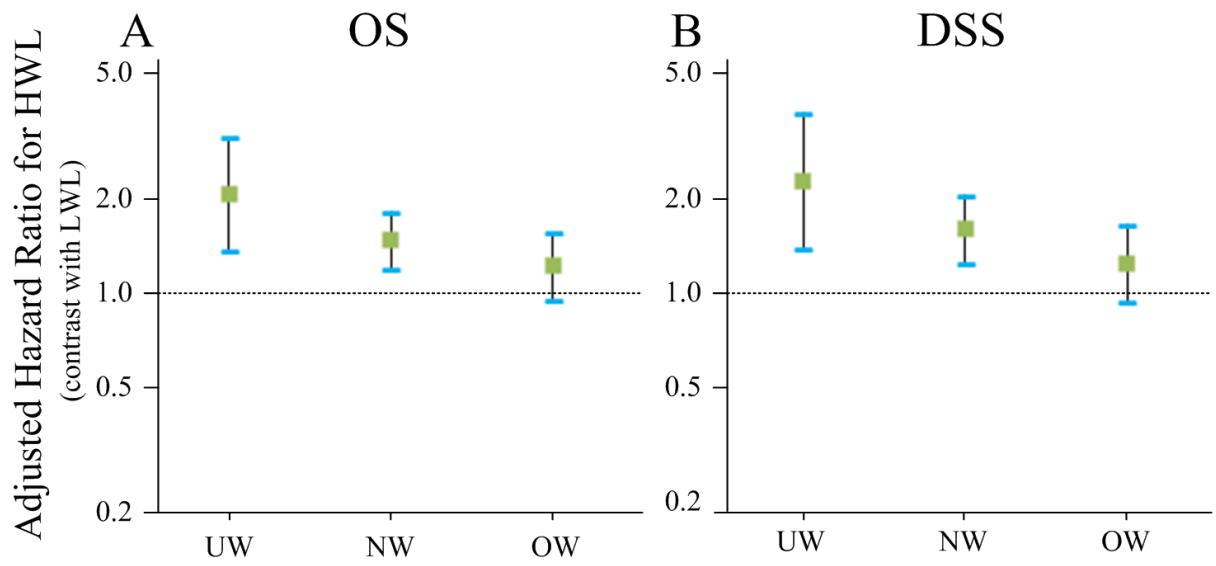

BMI Subgroups

Figure 3. Adjusted hazard ratios of high weight loss for survival in patients stratified by BMI levels. Hazard ratios of high weight loss for overall survival (OS) and disease-specific survival (DSS) in NPC patients receiving radical radiotherapy, stratified by BMI levels. HR was adjusted for age, sex, UICC T stage, UICC N stage, and treatment; $95 \%$ Cls were shown.

doi:10.1371/journal.pone.0068660.g003 overweight/obese $\times$ HWL vs underweight $\times$ LWL, $\mathrm{P}=0.054$; Table $\mathrm{S} 1)$. Therefore, we firstly divided the whole population into $2 \times 3$ weight-based subgroups according to BMI and weight loss. OS, DSS and LRFS were identified as significant in unadjusted analysis among the six subgroups, with joint $\mathrm{P}$ value $<0.001$ for all (Figure 2; Figure S1-S2); yet no significant difference was found for DMFS ( $\mathrm{P}=0.089)$.

Multiple-adjusted analysis including $2 \times 3$ weight-based subgroups, age, sex, UICC $\mathrm{T}$ stage, UICC $\mathrm{N}$ stage and treatment was performed. Comparing to NW+LWL patients, patients in UW+HWL group had significantly lower OS (HR, 2.56; 95\% CI 1.65-3.56), DSS (HR, 2.66; 95\% CI 1.82-3.88) and LRFS (HR, 2.62; 95\% CI 1.81-3.78); NW+HWL patients had mildly unfavorable OS (HR, 1.45; 95\% CI 1.18-1.78), DSS (HR, 1.57; 95\% CI 1.22-2.01) and LRFS (HR, 1.44; 95\% CI 1.14-1.82). By contrast, UW+LWL, OW+LWL, and OW+HWL patients did not have significantly different OS, DSS, or LRFS in comparison with NW+LWL patients (Table 3).

To assess the prognostic impact of weight loss for patients with different BMI levels, further stratified analysis was conducted (Table 4). Multiple-adjusted analysis showed HWL was independently associated with adverse OS (HR, 2.06; 95\% CI 1.36-3.11), DSS (HR, 2.27; 95\% CI 1.38-3.73), and LRFS (HR, 2.93; 95\% CI 1.75-4.90) among underweight patients. In the subgroup of normal weight patients, HWL was also significant on OS (HR, 1.47; 95\% CI 1.19-1.80), DSS (HR, 1.59; 95\% CI 1.24-2.03), and LRFS (HR, 1.45; 95\% CI 1.15-1.84). Among overweight/obese patients, no significant association between HWL and survival was found (Figure 3).

\section{Discussion}

$\mathrm{BMI}$ is one of the criteria commonly used in the assessment of nutritional status, whose prognostic impact has been extensively studied among NPG patients [6,7]. However, percentage weight loss, a sensitive and specific tool in assessment of recently developed malnutrition $[18,19]$, has been shown correlated with poor prognosis in head and neck cancer patients [11,20], and should be taken into account in NPG prognosis.

Currently, no data specifically focused on the prognostic impact of weight loss among NPC patients was available. In our series, it's 
Table 4. Outcomes according to weight loss stratified by BMI levels in multiple-adjusted Cox model adjusted for age, sex, UICC T stage, UICC N stage, and treatment.

\begin{tabular}{|c|c|c|c|c|c|c|c|c|c|}
\hline \multirow[t]{2}{*}{ Weight Change } & \multirow[t]{2}{*}{ No. } & \multicolumn{2}{|l|}{ os } & \multicolumn{2}{|l|}{ DSS } & \multicolumn{2}{|l|}{ LRFS } & \multicolumn{2}{|l|}{ DMFS } \\
\hline & & HR (95\% CI) & $\mathbf{P}$ & HR (95\% CI) & $\mathbf{P}$ & HR (95\% Cl) & $\mathbf{P}$ & HR (95\% CI) & $\mathbf{P}$ \\
\hline \multicolumn{10}{|c|}{ Underweight $(n=212)$} \\
\hline LWL & 131 & Ref & & Ref & & Ref & & Ref & \\
\hline HWL & 81 & $2.06(1.36-3.11)$ & 0.001 & $2.27(1.38-3.73)$ & 0.001 & $2.93(1.75-4.90)$ & $<0.001$ & $0.60(0.23-1.53)$ & 0.284 \\
\hline \multicolumn{10}{|c|}{ Normal weight $(\mathrm{n}=1157)$} \\
\hline LWL & 555 & Ref & & Ref & & Ref & & Ref & \\
\hline HWL & 602 & $1.47(1.19-1.80)$ & $<0.001$ & $1.59(1.24-2.03)$ & $<0.001$ & $1.45(1.15-1.84)$ & 0.002 & $1.15(0.74-1.78)$ & 0.537 \\
\hline \multicolumn{10}{|c|}{ Overweight/obese $(n=1064)$} \\
\hline LWL & 444 & Ref & & Ref & & Ref & & Ref & \\
\hline HWL & 620 & $1.22(0.95-1.55)$ & 0.117 & $1.23(0.93-1.64)$ & 0.153 & $1.23(0.93-1.63)$ & 0.146 & $0.92(0.55-1.54)$ & 0.761 \\
\hline
\end{tabular}

Footnote: Ref, reference; HR, hazard ratio. $\mathrm{P}<0.05$ was considered statistically significant.

doi:10.1371/journal.pone.0068660.t004

intriguing to find a potential differential impact of weight loss between underweight and overweight/obese patients $(\mathrm{P}=0.054)$. This indicates that a $2 \times 3$ grouping by BMI levels and weight loss and further stratified analysis by BMI levels are necessary to determine the impact of weight loss in NPC. Using the $2 \times 3$ grouping, we found that UW+HWL group had significantly lower OS comparing to the other five subgroups. Further stratified analysis by BMI levels showed HWL was independently associated with unfavorable OS and DSS among underweight patients (HR, 2.06; 95\% CI 1.36-3.11; HR, 2.27; 95\% CI 1.38-3.73). In normal weight patients, the impact of HWL was moderate (HR, 1.47; 95\% CI 1.19-1.80; HR, $1.59 ; 95 \%$ CI 1.24-2.03); among overweight/obese patients, such impact was weak (HR, 1.22; 95\% CI 0.95-1.55; HR, 1.23; 95\% CI 0.93-1.64). These novel observations underscore the importance of pretreatment BMI status in the interpretation of weight loss and, to our knowledge, represent the first data showing that high weight loss adversely impacts on survival in NPG patients.

The reason underlying the differential prognostic impact of weight loss on NPC patients of different BMI levels may mainly involve malnutrition. During the radiation treatment, underweight patients were more susceptible to severe side effects, such as mucositis, dysphagia, etc., than normal weight patients, which may affect their food intake and lead to body wasting [21-23]. Further high weight loss during the treatment may serve as a prominent indicator of malnutrition [20], which is known to be correlated with compromised immunity [23,24], unplanned treatment breaks and poor treatment outcomes in head and neck cancer [25-27]; by contrast, low weight loss in underweight patients may represent a well-tolerant nutritional status and is therefore associated with better prognosis $[7,28]$. The unfavorable impact of weight loss was moderate in normal weight patients, and even weaker in overweight/obese patients; some studies reported that NPC patients with normal or higher baseline BMI may be more resistance to malnutrition $[7,29,30]$, and this can partly explain our results. Apart from the impact of treatment, factors like advanced tumors, severe comorbidities and even insomnia can also contribute to body weight loss of NPG patients during radiation treatment [9]. Patients with advanced tumor are more likely to develop cancer cachexia syndrome, which is characterized by nutritionally irreversible weight loss and will greatly increase the mortality rate [31]. Comorbidities like diabetes, chronic obstructive pulmonary disease, and cardiovascular diseases will also worsen the prognosis of NPG patients and, on the other hand, exacerbate body wasting [32,33]. A recent study of 1001 newly diagnosed NPC patients showed that $15.5 \%$ elderly NPC patients ( $\geq 70$ years) presented with moderate to severe comorbidities (ACE-27 score $\geq 2$ ) before radiation treatment; these patients had lower OS (HR, 2.63, 95\% CI 1.45-4.76) than patients had none or mild comorbidities [33]. However, in our population, no patients presented with cachexia before treatment, and the proportion of elderly patients was rather small (16pts, $0.8 \%$ ). Therefore, this explanation may not be the main consideration.

Upon $2 \times 3$ grouping based on assortment of BMI and weight loss, we found UW+HWL patient group had lowest OS and DSS as compared with the other five groups, while e.g. the difference in survival rate between $\mathrm{UW}+\mathrm{LWL}$ and $\mathrm{NW}+\mathrm{LWL}$ patients was small (Figure 2). These data suggested that appropriate intervention towards weight loss, or malnutrition might be beneficial to underweight patients. For the past decade, there has been ample evidence that adequate nutrition support (NS) before and during radiotherapy can decrease the impact of side effect of treatment, minimize weight loss, and improve outcome for head and neck cancer patients $[34,35]$. Weight loss of more than $1-2 \%$ per week, or $5 \%$ in less than a month, should prompt further patient assessment, nutritional counseling, and more aggressive interventions [36]. However, Rabinovitch et al. analyzed the data of 1073 advanced HNC patients treated with definitive radiotherapy and found that patients with nutrition support during treatment had better 5-year locoregional control and 5-year overall survival rate as compared with patients receiving pre-treatment nutrition support, and that the pre-treatment nutritional support group even had poorer survival as compared with those with no nutritional support at all [37]. Therefore, how and when to adopt these nutritional interventions in NPG await further study.

Our study has several limitations. First, it is a retrospective study. Second, the proportion of obese patients in this study was relatively small $(6.5 \%)$, which might impede our interpretation of the prognostic value of HWL in this group; therefore, we merged the overweight and obese group. Third, the modes of chemotherapy applied varied, which might have a confounding effect. For these reasons, we need to validate our finding in a multiinstitutional prospective study in the future. 


\section{Conclusion}

Underweight NPC patients with high weight loss during radiation had worst survival compared with other subgroups assorted by BMI levels and weight loss. High weight loss is independently associated with increased mortality among underweight and normal weight, but not overweight/obese, NPC patients.

\section{Supporting Information}

Figure S1 DSS for NPG patients after radical radiotherapy in $2 \times 3$ grouping by BMI levels and weight loss status.

(TIF)

Figure S2 LRFS for NPG patients after radical radiotherapy in $2 \times 3$ grouping by BMI levels and weight loss status.

\section{References}

1. Lee AW, Poon YF, Foo W, Law SC, Cheung FK, et al. (1992) Retrospective analysis of 5037 patients with nasopharyngeal carcinoma treated during 1976 1985: overall survival and patterns of failure. Int J Radiat Oncol Biol Phys 23: 261-270.

2. Yu MC, Yuan JM (2002) Epidemiology of nasopharyngeal carcinoma. Semin Cancer Biol 12: 421-429.

3. Lee N, Harris J, Garden AS, Straube W, Glisson B, et al. (2009) Intensitymodulated radiation therapy with or without chemotherapy for nasopharyngeal carcinoma: radiation therapy oncology group phase II trial 0225. J Clin Oncol 27: $3684-3690$.

4. Chan AT, Teo PM, Johnson PJ (2002) Nasopharyngeal carcinoma. Ann Oncol 13: $1007-1015$.

5. Fang FM, Tsai WL, Chien CY, Chen HC, Hsu HC, et al. (2010) Pretreatment quality of life as a predictor of distant metastasis and survival for patients with nasopharyngeal carcinoma. J Clin Oncol 28: 4384-4389.

6. Hu JY, Yi W, Xia YF, Gao J, Liu ZG, et al. (2009) [Impact of pretherapy body mass index on prognosis of nasopharyngeal carcinoma]. Ai Zheng 28: 10431048 .

7. Shen GP, Xu FH, He F, Ruan HL, Cui C, et al. (2012) Pretreatment lifestyle behaviors as survival predictors for patients with nasopharyngeal carcinoma. PLoS One 7: e36515.

8. Ng K, Leung SF, Johnson PJ, Woo J (2004) Nutritional consequences of radiotherapy in nasopharynx cancer patients. Nutr Cancer 49: 156-161.

9. Qiu C, Yang N, Tian G, Liu H (2011) Weight loss during radiotherapy for nasopharyngeal carcinoma: a prospective study from northern China. Nutr Cancer 63: 873-879.

10. Kubrak C, Olson K, Jha N, Scrimger R, Parliament M, et al. (2012) Clinical determinants of weight loss in patients receiving radiation and chemoirradiation for head and neck cancer: A prospective longitudinal view. Head Neck.

11. Pai PC, Chuang CC, Tseng CK, Tsang NM, Chang KP, et al. (2012) Impact of pretreatment body mass index on patients with head-and-neck cancer treated with radiation. Int J Radiat Oncol Biol Phys 83: e93-e100.

12. Mao YP, Xie FY, Liu LZ, Sun Y, Li L, et al. (2009) Re-evaluation of 6th edition of AJCG staging system for nasopharyngeal carcinoma and proposed improvement based on magnetic resonance imaging. Int J Radiat Oncol Biol Phys 73: 1326-1334.

13. (2004) Appropriate body-mass index for Asian populations and its implications for policy and intervention strategies. Lancet 363: 157-163.

14. Beaver ME, Matheny KE, Roberts DB, Myers JN (2001) Predictors of weight loss during radiation therapy. Otolaryngol Head Neck Surg 125: 645-648.

15. van Bokhorst-de van der S, van Leeuwen PA, Kuik DJ, Klop WM, Sauerwein HP, et al. (1999) The impact of nutritional status on the prognoses of patients with advanced head and neck cancer. Cancer 86: 519-527.

16. Cheng SH, Yen KL, Jian JJ, Tsai SY, Chu NM, et al. (2001) Examining prognostic factors and patterns of failure in nasopharyngeal carcinoma following concomitant radiotherapy and chemotherapy: impact on future clinical trials. Int J Radiat Oncol Biol Phys 50: 717-726.

17. Sham JS, Choy D (1990) Prognostic factors of nasopharyngeal carcinoma: a review of 759 patients. Br J Radiol 63: 51-58.

18. Teitelbaum D, Guenter P, Howell WH, Kochevar ME, Roth J, et al. (2005) Definition of terms, style, and conventions used in A.S.P.E.N. guidelines and standards. Nutr Clin Pract 20: 281-285.

19. Ravasco P, Monteiro-Grillo I, Vidal PM, Camilo ME (2003) Nutritional deterioration in cancer: the role of disease and diet. Clin Oncol (R Coll Radiol) 15: $443-450$.

20. Datema FR, Ferrier MB, Baatenburg de Jong RJ (2011) Impact of severe malnutrition on short-term mortality and overall survival in head and neck cancer. Oral Oncol 47: 910-914.
(TIF)

Table S1 Test for interaction between weight loss and BMI levels, $\mathbf{T}$ stage, $\mathbf{N}$ stage, age and treatment on $O S$. (DOCX)

\section{Acknowledgments}

We would like to thank Nan-Qiao Cai for providing support on statistics, and Li-Shan Zeng, Nan Wu and Tom Liu for critically reading and editing this manuscript.

\section{Author Contributions}

Conceived and designed the experiments: LJS CG BFL JG YFX. Performed the experiments: LJS CG BFL JG YFX. Analyzed the data: LJS CG BFL JG YFX. Contributed reagents/materials/analysis tools: LJS CG BFL YFX. Wrote the paper: LJS YFX.

21. Salas S, Deville JL, Giorgi R, Pignon T, Bagarry D, et al. (2008) Nutritional factors as predictors of response to radio-chemotherapy and survival in unresectable squamous head and neck carcinoma. Radiother Oncol 87: 195200.

22. Meyer F, Fortin A, Wang CS, Liu G, Bairati I (2012) Predictors of severe acute and late toxicities in patients with localized head-and-neck cancer treated with radiation therapy. Int J Radiat Oncol Biol Phys 82: 1454-1462.

23. O'Shea D, Cawood TJ, O'Farrelly C, Lynch L, WHO Expert Consultation (2010) Natural killer cells in obesity: impaired function and increased susceptibility to the effects of cigarette smoke. PLoS One 5: e8660.

24. He JR, Shen GP, Ren ZF, Oin H, Cui C, et al. (2012) Pretreatment levels of peripheral neutrophils and lymphocytes as independent prognostic factors in patients with nasopharyngeal carcinoma. Head Neck.

25. Kwong DL, Sham JS, Chua DT, Choy DT, Au GK, et al. (1997) The effect of interruptions and prolonged treatment time in radiotherapy for nasopharyngeal carcinoma. Int J Radiat Oncol Biol Phys 39: 703-710.

26. Capuano G, Grosso A, Gentile PC, Battista M, Bianciardi F, et al. (2008) Influence of weight loss on outcomes in patients with head and neck cancer undergoing concomitant chemoradiotherapy. Head Neck 30: 503-508.

27. Ghadjar P, Simcock M, Zimmermann F, Betz M, Bodis S, et al. (2012) Predictors of severe late radiotherapy-related toxicity after hyperfractionated radiotherapy with or without concomitant cisplatin in locally advanced head and neck cancer. Secondary retrospective analysis of a randomized phase III trial (SAKK 10/94). Radiother Oncol 104: 213-218.

28. Nourissat A, Bairati I, Samson E, Fortin A, Gelinas M, et al. (2010) Predictors of weight loss during radiotherapy in patients with stage I or II head and neck cancer. Cancer 116: 2275-2283.

29. Li G, Gao J, Liu ZG, Tao YL, Xu BO, et al. (2013) Influence of pretreatment ideal body weight percentile and albumin on the prognosis of nasopharyngeal carcinoma: Long-term outcomes of 512 patients from a single institution. Head Neck.

30. Huang PY, Wang CT, Cao KJ, Guo X, Guo L, et al. (2013) Pretreatment body mass index as an independent prognostic factor in patients with locoregionally advanced nasopharyngeal carcinoma treated with chemoradiotherapy: Findings from a randomised trial. Eur J Cancer 49: 1923-1931.

31. Couch M, Lai V, Cannon T, Guttridge D, Zanation A, et al. (2007) Cancer cachexia syndrome in head and neck cancer patients: part I. Diagnosis, impact on quality of life and survival, and treatment. Head Neck 29: 401-411.

32. Sze HC, Ng WT, Chan OS, Shum TC, Chan LL, et al. (2012) Radical radiotherapy for nasopharyngeal carcinoma in elderly patients: the importance of co-morbidity assessment. Oral Oncol 48: 162-167.

33. Ramakrishnan Y, Paleri V, Shah R, Steen IN, Wight RG, et al. (2007) Comorbidity in nasopharyngeal carcinoma: a preliminary communication on the prevalence, descriptive distribution and impact on outcome. Clin Otolaryngol 32: 484-488.

34. Isenring EA, Capra S, Bauer JD (2004) Nutrition intervention is beneficial in oncology outpatients receiving radiotherapy to the gastrointestinal or head and neck area. Br J Cancer 91: 447-452.

35. Canada T (2002) Clinical dilemma in cancer: is tumor growth during nutrition support significant? Nutr Clin Pract 17: 246-248.

36. Larsson M, Hedelin B, Johansson I, Athlin E (2005) Eating problems and weight loss for patients with head and neck cancer: a chart review from diagnosis until one year after treatment. Cancer Nurs 28: 425-435.

37. Rabinovitch R, Grant B, Berkey BA, Raben D, Ang KK, et al. (2006) Impact of nutrition support on treatment outcome in patients with locally advanced head and neck squamous cell cancer treated with definitive radiotherapy: a secondary analysis of RTOG trial 90-03. Head Neck 28: 287-296. 